\title{
A STUDY ON THE PARA-HOLOMORPHIC SECTIONAL CURVATURE OF PARA-KÄHLER COTANGENT BUNDLES
}

\author{
BY
}

\section{S. L. DRUŢĂ-ROMANIUC}

\begin{abstract}
We obtain the conditions under which the total space $T^{*} M$ of the cotangent bundle, endowed with a natural diagonal para-Kähler structure $(G, P)$, has constant para-holomorphic sectional curvature. Moreover we prove that $\left(T^{*} M, G, P\right)$ cannot have nonzero constant para-holomorphic sectional curvature.

Mathematics Subject Classification 2010: 53C05, 53C15, 53C55.

Key words: natural lift, para-Hermitian structure, para-Kähler structure, paraholomorphic sectional curvature.
\end{abstract}

\section{Introduction}

Considering natural lifts of the Riemannian metric $g$ from the base manifold $M$ to the total space $T^{*} M$ of the cotangent bundle, some new interesting geometric structures were studied e.g. in [7]-[11], [19]-[23], [25]. The lifts to $T^{*} M$ cannot be constructed in the same manner as the lifts to the total space $T M$ of the tangent bundle (see [26]), and this fact explains the fundamental differences between the geometry of the two dual bundles.

Some almost para-Hermitian structures (called also almost hyperbolic Hermitian structures) were constructed on the total spaces of the tangent and cotangent bundles, in papers such as [2]-[6], [11]-[15] and [23].

BEJAN classified the almost para-Hermitian manifolds, obtaining in [2] 36 classes, up to duality. GADEA and MUÑOZ-MASQUÉ gave a classification à la Gray-Hervella, obtaining in [12] 136 classes, up to duality. Maybe the best known class of (almost) para-Hermitian manifolds, characterized by the 
closedness of the associated 2-form, is the class of the (almost) para-Kähler manifolds, studied for example in [1].

PRVANOVIC introduced in [24] the notion of para-holomorphic sectional curvature of a para-Kähler manifold, analogously to the holomorphic sectional curvature of a Kähler manifold. She also introduced the para-holomorphic projective curvature tensor, or H-projectiv curvature tensor, and she obtained the explicit expression of the curvature tensor field for spaces with constant para-holomorphic sectional curvature, studied e.g. in [13].

In the present paper, we get the conditions under which the natural diagonal para-Kähler cotangent bundle $\left(T^{*} M, G, P\right)$, characterized in [11], has constant para-holomorphic sectional curvature. Moreover, we prove that under the obtained conditions, the constant para-holomorphic sectional curvature vanishes.

Throughout this paper, the manifolds, tensor fields and other geometric objects are assumed to be differentiable of class $C^{\infty}$ (i.e. smooth). The Einstein summation convention is used, the range of the indices $h, i, j, k, l, m, r$, being always $\{1, \ldots, n\}$.

\section{Preliminary results}

The cotangent bundle of a smooth $n$-dimensional Riemannian manifold may be endowed with a structure of $2 n$-dimensional smooth manifold, induced by the structure on the base manifold. If $(M, g)$ is a smooth Riemannian manifold of dimension $n$, we denote its cotangent bundle by $\pi: T^{*} M \rightarrow M$. Every local chart on $M,(U, \varphi)=\left(U, x^{1}, \ldots, x^{n}\right)$ induces a local chart $\left(\pi^{-1}(U), \Phi\right)=\left(\pi^{-1}(U), q^{1}, \ldots, q^{n}, p_{1}, \ldots, p_{n}\right)$, on $T^{*} M$, as follows. For a cotangent vector $p \in \pi^{-1}(U) \subset T^{*} M$, the first $n$ local coordinates $q^{1}, \ldots, q^{n}$ are the local coordinates of its base point $x=\pi(p)$ in the local chart $(U, \varphi)$ (in fact we have $q^{i}=\pi^{*} x^{i}=x^{i} \circ \pi, i=1, \ldots n$ ). The last $n$ local coordinates $p_{1}, \ldots, p_{n}$ of $p \in \pi^{-1}(U)$ are the vector space coordinates of $p$ with respect to the basis $\left(d x_{\pi(p)}^{1}, \ldots, d x_{\pi(p)}^{n}\right)$, defined by the local chart $(U, \varphi)$, i.e. $p=p_{i} d x_{\pi(p)}^{i}$.

The concept of $M$-tensor field on the cotangent bundle of a Riemannian manifold, recalled in [7], can be introduced the same manner as the $M$ tensor fields on the tangent bundle (see [18]).

We recall the splitting of the tangent bundle to $T^{*} M$ into the vertical distribution $V T^{*} M=\operatorname{Ker} \pi_{*}$ and the horizontal one, determined by the 
Levi Civita connection $\dot{\nabla}$ of the metric $g$ :

$$
T T^{*} M=V T^{*} M \oplus H T^{*} M .
$$

If $\left(\pi^{-1}(U), \Phi\right)=\left(\pi^{-1}(U), q^{1}, \ldots, q^{n}, p_{1}, \ldots, p_{n}\right)$ is a local chart on $T^{*} M$, induced from the local chart $(U, \varphi)=\left(U, x^{1}, \ldots, x^{n}\right)$, the local vector fields $\frac{\partial}{\partial p_{1}}, \ldots, \frac{\partial}{\partial p_{n}}$ on $\pi^{-1}(U)$ define a local frame for $V T^{*} M$ over $\pi^{-1}(U)$ and the local vector fields $\frac{\delta}{\delta q^{1}}, \ldots, \frac{\delta}{\delta q^{n}}$ define a local frame for $H T^{*} M$ over $\pi^{-1}(U)$, where $\frac{\delta}{\delta q^{i}}=\frac{\partial}{\partial q^{i}}+\Gamma_{i h}^{0} \frac{\partial}{\partial p_{h}}, \Gamma_{i h}^{0}=p_{k} \Gamma_{i h}^{k}$, and $\Gamma_{i h}^{k}(\pi(p))$ are the Christoffel symbols of $g$.

The set of vector fields $\left\{\frac{\partial}{\partial p_{i}}, \frac{\delta}{\delta q^{j}}\right\}_{i, j=\overline{1, n}}$, denoted by $\left\{\partial^{i}, \delta_{j}\right\}_{i, j=\overline{1, n}}$, defines a local frame on $T^{*} M$, adapted to the direct sum decomposition (2.1).

We consider the energy density defined by $g$ in the cotangent vector $p$ :

$$
t=\frac{1}{2}\|p\|^{2}=\frac{1}{2} g_{\pi(p)}^{-1}(p, p)=\frac{1}{2} g^{i k}(x) p_{i} p_{k}, \quad p \in \pi^{-1}(U) .
$$

We have $t \in[0, \infty)$ for all $p \in T^{*} M$.

\section{The constant para-holomorphic sectional curvature of the natural diagonal para-Kähler cotangent bundles}

In this section we shall determine the conditions under which the paraKähler manifold ( $\left.T^{*} M, G, P\right)$ obtained in [11] has constant para-holomorphic sectional curvature.

Now we recall some results obtained in [11], concerning the natural diagonal almost product (locally product) and (almost) para-Hermitian structures on the (total space of the) cotangent bundle, and in particular the natural diagonal (almost) para-Kähler structures on $T^{*} M$.

An almost product structure $J$ on a differentiable manifold $M$ is a $(1,1)$ tensor field on $M$ such that $J^{2}=I$. The pair $(M, J)$ is called an almost product manifold. When the almost product structure $J$ is integrable, it is called a locally product structure, and $(M, J)$ is a locally product manifold.

The results from [16] and [17] concerning the natural lifts, allowed us to introduce in $[11]$ a $(1,1)$ tensor field $P$ on $T^{*} M$, which is a natural diagonal lift of the metric $g$ from the base manifold to the cotangent bundle. Using the adapted frame $\left\{\partial^{i}, \delta_{j},\right\}_{i, j=\overline{1, n}}$ to $T^{*} M$, we locally define $P$ by the relations:

$$
P \delta_{i}=P_{i j}^{(1)} \partial^{j}, \quad P \partial^{i}=P_{(2)}^{i j} \delta_{j}
$$


where the $M$-tensor fields involved as coefficients have the forms

$$
P_{i j}^{(1)}=a_{1}(t) g_{i j}+b_{1}(t) p_{i} p_{j}, \quad P_{(2)}^{i j}=a_{2}(t) g^{i j}+b_{2}(t) g^{0 i} g^{0 j}
$$

$a_{1}, b_{1}, a_{2}$, and $b_{2}$ being smooth functions of the energy density $t$.

The invariant expression of the defined structure is

$P X_{p}^{H}=a_{1}(t)\left(X^{b}\right)_{p}^{V}+b_{1}(t) p(X) p_{p}^{V}, P \alpha_{p}^{V}=a_{2}(t)\left(\alpha^{\sharp}\right)_{p}^{H}+b_{2}(t) g_{\pi(p)}^{-1}(p, \alpha)\left(p^{\sharp}\right)_{p}^{H}$,

in every point $p$ of the induced local card $\left(\pi^{-1}(U), \Phi\right)$ on $T^{*} M, \forall X \in$ $\mathcal{T}_{0}^{1}(M), \forall \alpha \in \mathcal{T}_{1}^{0}(M)$. The vector $p^{\sharp}$ is tangent to $M$ in $\pi(p), p^{V}=p_{i} \partial^{i}$ is the Liouville vector field on $T^{*} M$, and $\left(p^{\sharp}\right)^{H}=g^{0 i} \delta_{i}$ is the geodesic spray.

In the sequel we shall recall the conditions under which the above tensor field $P$ defines an almost product (locally product) structure on the total space of the cotangent bundle (see [11]).

The tensor field $P$, given by (3.1), defines an almost product structure of natural diagonal lift type on $T^{*} M$, if and only if its coefficients satisfy

$$
a_{1}=1 / a_{2}, \quad a_{1}+2 t b_{1}=1 /\left(a_{2}+2 t b_{2}\right) .
$$

The natural diagonal almost product structure $P$ is a locally product structure on $T^{*} M$ (i.e. $P$ is integrable) if and only if the base manifold is of constant sectional curvature $c$, and the coefficients $b_{1}, b_{2}$ are given by:

$$
b_{1}=\left(a_{1} a_{1}^{\prime}+c\right) /\left(a_{1}-2 t a_{1}^{\prime}\right), b_{2}=\left(a_{1} a_{2}^{\prime}-a_{2}^{2} c\right) /\left(a_{1}+2 c t a_{2}\right) .
$$

A Riemannian manifold $(M, g)$, endowed with an almost product structure $J$, satisfying the relation

$$
g(J X, J Y)=-g(X, Y), \forall X, Y \in \mathcal{T}_{0}^{1}(M),
$$

is called a an almost para-Hermitian manifold (or an almost hyperbolic Hermitian manifold).

We considered in [11] a natural diagonal lifted metric on the total space $T^{*} M$ of the cotangent bundle, defined by:

$$
\left\{\begin{array}{l}
G_{p}\left(X^{H}, Y^{H}\right)=c_{1}(t) g_{\pi(p)}(X, Y)+d_{1}(t) p(X) p(Y) \\
G_{p}\left(\alpha^{V}, \omega^{V}\right)=c_{2}(t) g_{\pi(p)}^{-1}(\alpha, \omega)+d_{2}(t) g_{\pi(p)}^{-1}(p, \alpha) g_{\pi(p)}^{-1}(p, \omega), \\
G_{p}\left(X^{H}, \alpha^{V}\right)=G_{p}\left(\alpha^{V}, X^{H}\right)=0
\end{array}\right.
$$


$\forall X, Y \in \mathcal{T}_{0}^{1}(M), \forall \alpha, \omega \in \mathcal{T}_{1}^{0}(M), \forall p \in T^{*} M$, where the coefficients $c_{1}, c_{2}$, $d_{1}, d_{2}$ are smooth functions of the energy density.

The conditions for $G$ to be nondegenerate are assured if

$$
c_{1} c_{2} \neq 0,\left(c_{1}+2 t d_{1}\right)\left(c_{2}+2 t d_{2}\right) \neq 0 .
$$

The metric $G$ is positive definite if and only if $c_{1}+2 t d_{1}>0, c_{2}+2 t d_{2}>0$.

With respect the adapted frame on $T^{*} M$, (3.6) can be written in the form

$$
\left\{\begin{array}{l}
G\left(\delta_{i}, \delta_{j}\right)=G_{i j}^{(1)}=c_{1}(t) g_{i j}+d_{1}(t) p_{i} p_{j} \\
G\left(\partial^{i}, \partial^{j}\right)=G_{(2)}^{i j}=c_{2}(t) g^{i j}+d_{2}(t) g^{0 i} g^{0 j} \\
G\left(\partial^{i}, \delta_{j}\right)=G\left(\delta_{i}, \partial^{j}\right)=0
\end{array}\right.
$$

where $c_{1}, c_{2}, d_{1}, d_{2}$ are smooth functions of the density energy on $T^{*} M$.

Next we proved that $\left(T^{*} M, G, P\right)$ is a para-Hermitian manifold if and only if the integrability conditions for $P$ and the following relations hold good:

$$
\frac{c_{1}}{a_{1}}=-\frac{c_{2}}{a_{2}}=\lambda, \frac{c_{1}+2 t d_{1}}{a_{1}+2 t b_{1}}=-\frac{c_{2}+2 t d_{2}}{a_{2}+2 t b_{2}}=\lambda+2 t \mu,
$$

where the proportionality coefficients $\lambda>0$ and $\lambda+2 t \mu>0$ are some functions depending on the energy density $t$.

Moreover, the para-Hermitian manifold $\left(T^{*} M, G, P\right)$ is para-Kähler (i.e. the associated 2-form $\Omega(X, Y)=G(X, P Y), \forall X, Y \in \mathcal{T}_{0}^{1}\left(T^{*} M\right)$, is closed) if and only if $\mu=\lambda^{\prime}$.

Remark 1. The natural diagonal para-Kähler structures on $T^{*} M$ depend on two essential coefficients, $a_{1}$ and $\lambda$, which must satisfy the conditions $a_{1}>0, a_{1}+2 t b_{1}>0, \lambda>0, \lambda+2 t \lambda^{\prime}>0$, where $b_{1}$ is given by (3.4).

Adapting [8, Theorem 3.1] to the context of the natural diagonal lifted metric on $T^{*} M$, we obtain the following:

Proposition 3.1. The Levi-Civita connection $\nabla$ of the natural diagonal lifted metric $G$ on the cotangent bundle of the Riemannian manifold $(M, g)$ has the following expression with respect to the local adapted frame $\left\{\delta_{i}, \partial^{j}\right\}_{i, j=1, \ldots, n}$ :

$$
\left\{\begin{array}{l}
\nabla_{\partial^{i}} \partial^{j}=Q^{i j}{ }_{h} \partial^{h}, \nabla_{\delta_{i}} \partial^{j}=-\Gamma_{i h}^{j} \partial^{h}+P_{i}{ }^{h j} \delta_{h} \\
\nabla_{\partial^{i}} \delta_{j}=P_{j}{ }^{i h} \delta_{h}, \nabla_{\delta_{i}} \delta_{j}=\Gamma_{i j}^{h} \delta_{h}+S_{i j h} \partial^{h}
\end{array}\right.
$$


where $\Gamma_{i j}^{h}$ are the Christoffel symbols of the Levi-Civita connection $\dot{\nabla}$ on the base manifold, and the $M$-tensor fields involved as coefficients are given by

$$
\left\{\begin{array}{l}
Q^{i j}{ }_{h}=\frac{1}{2}\left(\partial^{i} G_{(2)}^{j k}+\partial^{j} G_{(2)}^{i k}-\partial^{k} G_{(2)}^{i j}\right) H_{k h}^{(2)}, \\
P_{j}{ }^{i h}=\frac{1}{2}\left(\partial^{i} G_{j k}^{(1)}-R_{l j k}^{0} G_{(2)}^{l i}\right) H_{(1)}^{k h} \\
S_{i j h}=\frac{1}{2}\left(R_{h i j}^{0}-\partial^{k} G_{i j}^{(1)} H_{k h}^{(2)}\right)
\end{array}\right.
$$

$R_{k i j}^{h}$ being the components of the curvature tensor field of $\dot{\nabla}$.

The curvature tensor field $K$ of the para-Kähler manifold $\left(T^{*} M, G, P\right)$ has the following components with respect to the adapted frame $\left\{\delta_{i}, \partial^{j}\right\}_{i, j=1, \ldots, n}$ :

$$
\begin{aligned}
& K\left(\delta_{i}, \delta_{j}\right) \delta_{k}=Q Q Q Q_{i j k}{ }^{h} \delta_{h}=\left(P_{i}{ }^{l h} S_{j k l}-P_{j}{ }^{l h} S_{i k l}-R_{l i j}^{0} P_{k}{ }^{l h}+R_{k i j}^{h}\right) \delta_{h}, \\
& K\left(\delta_{i}, \delta_{j}\right) \partial^{k}=Q Q P P_{i j}{ }_{h}{ }_{h} \partial^{h}=\left(P_{j}{ }^{k l} S_{i l h}-P_{i}{ }^{k l} S_{j l h}-R_{l i j}^{0} Q^{l k}{ }_{h}-R_{h i j}^{k}\right) \partial^{h}, \\
& K\left(\partial^{i}, \partial^{j}\right) \delta_{k}=P P Q Q^{i j}{ }_{k}{ }^{h} \delta_{h}=\left(\partial^{i} P_{k}{ }^{j h}-\partial^{j} P_{k}{ }^{i h}+P_{k}{ }^{j l} P_{l}{ }^{i h}-P_{k}{ }^{i l} P_{l}{ }^{j h}\right) \delta_{h}, \\
& K\left(\partial^{i}, \partial^{j}\right) \partial^{k}=P P P P^{i j k}{ }_{h} \partial^{h}=\left(\partial^{i} Q^{j k}{ }_{h}-\partial^{j} Q^{i k}{ }_{h}+Q^{j k}{ }_{l} Q^{i l}{ }_{h}-Q^{i k}{ }_{l} Q^{j l}{ }_{h}\right) \partial^{h}, \\
& K\left(\partial^{i}, \delta_{j}\right) \delta_{k}=P Q Q P^{i}{ }_{j k h} \partial^{h}=\left(\partial^{i} S_{j k h}+S_{j k l} Q^{i l}{ }_{h}-S_{j l h} P_{k}{ }_{k}{ }^{l l}\right) \partial^{h}, \\
& K\left(\partial^{i}, \delta_{j}\right) \partial^{k}=P Q P Q^{i}{ }_{j}{ }^{k h} \delta_{h}=\left(\partial^{i} P_{j}{ }^{k h}+P_{l}{ }^{i h} P_{j}{ }^{k l}-Q^{i k}{ }_{l} P_{j}{ }^{l h}\right) \delta_{h},
\end{aligned}
$$

In [11] we proved that the above components are of the form

$$
\begin{gathered}
\alpha_{1} g_{h j} g^{i k}+\alpha_{2} \delta_{h}^{i} \delta_{j}^{k}+\alpha_{3} \delta_{h}^{k} \delta_{j}^{i}+\alpha_{4} g^{i k} p_{h} p_{j} \alpha_{5} \delta_{j}^{k} p_{h} g^{0 i}+\alpha_{6} \delta_{h}^{k} p_{j} g^{0 i}+ \\
+\alpha_{7} \delta_{j}^{i} p_{h} g^{0 k}+\alpha_{8} g_{h j} g^{0 i} g^{0 k}+\alpha_{9} \delta_{h}^{i} p_{j} g^{0 k}+\alpha_{10} p_{h} p_{j} g^{0 i} g^{0 k}
\end{gathered}
$$

where the coefficients $\alpha_{1}, \ldots, \alpha_{10}$ are smooth functions on $T^{*} M$, depending on $a_{1}, \lambda$, their first three orders derivatives, the energy density, and the constant sectional curvature $c$ of the base manifold. In a few components of $K$, some of the coefficients $\alpha_{1}, \ldots, \alpha_{10}$ vanish.

In [24] PRVANOvic defined the curvature tensor field of a para-Kähler manifold of constant para-holomorphic sectional curvature, $k$. In the case of the para-Kähler manifold $\left(T^{*} M, G, P\right)$, we obtain the expression:

$$
\begin{aligned}
K_{0}(X, Y) Z & =\frac{k}{4}[G(Y, Z) X-G(X, Z) Y+G(Y, P Z) P X \\
& -G(X, P Z) P Y-2 G(X, P Y) P Z], \quad \forall X, Y, Z \in \mathcal{T}_{0}^{1}\left(T^{*} M\right),
\end{aligned}
$$


and the components with respect to the adapted frame $\left\{\delta_{i}, \partial^{j}\right\}_{i, j=1, \ldots, n}$ are

$$
\begin{aligned}
K_{0}\left(\delta_{i}, \delta_{j}\right) \delta_{k} & =\frac{k}{4}\left(G_{j k}^{(1)} \delta_{i}^{h}-G_{i k}^{(1)} \delta_{j}^{h}\right) \delta_{h}, \\
K_{0}\left(\delta_{i}, \delta_{j}\right) \partial^{k} & =\frac{k}{4}\left(G_{j l}^{(1)} P_{i h}^{(1)}-G_{i l}^{(1)} P_{j h}^{(1)}\right) P_{(2)}^{k l} \partial^{h}, \\
K_{0}\left(\partial^{i}, \partial^{j}\right) \delta_{k} & =\frac{k}{4}\left(G_{(2)}^{j l} P_{(2)}^{i h}-G_{(2)}^{i l} P_{(2)}^{j h}\right) P_{k l}^{(1)} \delta_{h}, \\
K_{0}\left(\partial^{i}, \partial^{j}\right) \partial^{k} & =\frac{k}{4}\left(G_{(2)}^{j k} \delta_{h}^{i}-G_{(2)}^{i k} \delta_{h}^{j}\right) \partial^{h} \\
K_{0}\left(\partial^{i}, \delta_{j}\right) \delta_{k} & =\frac{k}{4}\left[G_{j k}^{(1)} \delta_{h}^{i}-G_{(2)}^{i l}\left(P_{k l}^{(1)} P_{j h}^{(1)}-2 P_{j l}^{(1)} P_{k h}^{(1)}\right)\right] \partial^{h}, \\
K_{0}\left(\partial^{i}, \delta_{j}\right) \partial^{k} & =\frac{k}{4}\left(-G_{(2)}^{i k} \delta_{j}^{h}+G_{j l}^{(1)} P_{(2)}^{k l} P_{(2)}^{i h}-2 G_{(2)}^{i l} P_{j l}^{(1)} P_{(2)}^{k h}\right) \delta_{h} .
\end{aligned}
$$

The para-Kähler manifold $\left(T^{*} M, G, P\right)$ has constant para-holomorphic sectional curvature if and only if the equality $K=K_{0}$ is satisfied. So, we have to study the conditions of vanishing for all the components of the difference $K-K_{0}$. To this aim, we shall use the following result, provided in $[7]$.

Lemma 3.2. If $\alpha_{1}, \ldots, \alpha_{10}$ are smooth functions on $T^{*} M$ such that the relation (3.10) is satisfied, then $\alpha_{1}=\cdots=\alpha_{10}=0$.

Next we shall prove the characterization theorem for the natural diagonal para-Kähler cotangent bundles of constant para-holomorphic sectional curvature.

Theorem 3.3. The natural diagonal para-Kähler manifold $\left(T^{*} M, G, P\right)$, characterized in [11], has constant para-holomorphic sectional curvature $k$, if and only if the constant sectional curvature $c$ of the base manifold and the essential coefficients mentioned in Remark 1 satisfy the following conditions: $c=0$ (i.e. the base manifold $M$ is flat) and

$$
a_{1}^{\prime \prime}=\frac{4 a_{1}^{\prime 2}\left(a_{1}-a_{1}^{\prime} t\right)}{a_{1}\left(a_{1}-2 a_{1}^{\prime} t\right)}, c_{1}=\mathcal{C}, \lambda=\frac{\mathcal{C}}{a_{1}},
$$

where $\mathcal{C}$ is a real constant. Moreover, the para-Kähler manifold $\left(T^{*} M, G, P\right)$ of natural diagonal lift type cannot have nonzero constant para-holomorphic sectional curvature. 
Proof. The components of $K-K_{0}$ with respect to the adapted frame $\left\{\delta_{i}, \partial^{j}\right\}_{i, j=1, \ldots, n}$ are combinations of the form (3.10), hence in the study of their vanishing conditions, we can apply Lemma 3.2. Thus, analyzing the terms of the component $\left(K-K_{0}\right)\left(\partial^{i}, \delta_{j}\right) \partial^{k}$, we have that the term containing $g^{h k} \delta_{j}^{i}$ vanishes if and only if the derivative of the proportionality factor $\lambda$ has the expression

$$
\lambda^{\prime}=\lambda \frac{a_{1} k\left(a_{1}-2 t a_{1}^{\prime}\right) \lambda-a_{1}^{2} a_{1}^{\prime}-2 c\left(a_{1}-t a_{1}^{\prime}\right)}{a_{1}\left(a_{1}^{2}+2 c t\right)} .
$$

Imposing the conditions of vanishing for the coefficients of $g^{h i} \delta_{j}^{k}$ and $g^{i k} \delta_{j}^{h}$, and taking into account the relation (3.12) we obtain the equations $-4 a_{1} c+a_{1}^{2} k \lambda-2 c k \lambda t=0, \quad 4 a_{1} c-3 a_{1}^{2} k \lambda+6 c k \lambda t-4 a_{1} k^{2} \lambda^{2} t=0$, from which we have that $k=\frac{4 a_{1} c}{\lambda\left(a_{1}^{2}-2 c t\right)}$, and then

$$
-8 a_{1} c\left(a_{1}^{2}+2 c t\right)^{2} /\left(a_{1}^{2}-2 c t\right)^{2}=0 .
$$

Since $a_{1} a_{2}=1, a_{1}$ cannot vanish. Hence (3.13) leads to the following cases:

Case I) $a_{1}^{2}=-2$ ct may be discussed only when the base manifold is of negative constant sectional curvature. In this situation we obtain $a_{1}=$ $2 t a_{1}^{\prime}$, which cannot hold, since $a_{1}-2 t a_{1}^{\prime}$ appears as a denominator in the integrability conditions (3.4).

Case $I I) c=0$ (i.e. the base manifold is flat) leads to $k=0$ too. From (3.12) we obtain the differential equation $\lambda^{\prime} / \lambda=-a_{1}^{\prime} / a_{1}$, with the solution $\lambda=\frac{\mathcal{C}}{a_{1}}$, where $\mathcal{C}$ is a real constant.

Replacing the above value of $\lambda$ into (3.8), we obtain that $\frac{c_{1}}{a_{1}}=\frac{\mathcal{C}}{a_{1}}$, so the coefficient $c_{1}$ involved in the definition of the metric $G$ is a real constant.

By substituting the value of $\lambda$, and taking into account that $c=k=0$, we obtain that all the components of $K-K_{0}$ vanish, except $\left(K-K_{0}\right)\left(\partial^{i}, \partial^{j}\right) \partial^{k}$, which becomes

$$
\begin{aligned}
& 2 \frac{a_{1} a_{1}^{\prime \prime}\left(a_{1}-2 a_{1}^{\prime} t\right)+4 a_{1}^{\prime 2}\left(a_{1}^{\prime} t-a_{1}\right)}{a_{1}-2 a_{1}^{\prime} t}\left[\frac{1}{\left(a_{1}-2 a_{1}^{\prime} t\right)^{2}}\left(g^{j k} g^{0 i}-g^{i k} g^{0 j}\right) p_{h}\right. \\
& \left.+\frac{1}{a_{1}^{2}}\left(\delta_{h}^{i} g^{0 j}-\delta_{h}^{j} g^{0 i}\right) g^{0 k}\right] \partial^{h}
\end{aligned}
$$

and which vanishes if and only if $a_{1}$ satisfy the differential equation in (3.11). 
Acknowledgements. The author expresses her gratitude to professor V. Oproiu for the valuable suggestions throughout this work.

\section{REFERENCES}

1. Alekseevsky, D.V.; Medori, K.; Tomassini, A. - Homogeneous para-Kählerian Einstein manifolds, (Russian) Uspekhi Mat. Nauk, 64 (2009), 3-50; translation in Russian Math. Surveys 64 (2009), 1-43.

2. Bejan, C. - A classification of the almost para-Hermitian manifolds, Differential geometry and its applications (Dubrovnik, 1988), 23-27, Univ. Novi Sad, Novi Sad, 1989.

3. Bejan, C.-L. - Almost para-Hermitian structures on the tangent bundle of an almost para-co-Hermitian manifold, The Proceedings of the Fifth National Seminar of Finsler and Lagrange Spaces (Braşov, 1988), 105-109, Soc. Ştiinţe Mat. R.S. România, Bucharest, 1989.

4. BeJAn, C.-L. - Some examples of manifolds with hyperbolic structures, Rend. Mat. Appl., 14 (1994), 557-565.

5. Bejan, C.-L.; OrneA, L. - An example of an almost hyperbolic Hermitian manifold, Internat. J. Math. Math. Sci., 21 (1998), 613-618.

6. Cruceanu, V. - Selected Papers, Editura PIM, Iaşi, 2006, (37. Para-Hermitian and para-Kähler manifolds, pp. 339-387.)

7. Druţă, S.L. - Cotangent bundles with general natural Kähler structures, Rev. Roumaine Math. Pures Appl., 54 (2009), 13-23.

8. DRUţă, S.L. - The holomorphic sectional curvature of general natural Kähler structures on cotangent bundles, An. Ştiinţ. Univ. "Al. I. Cuza" Iaşi. Mat. (N.S.), 56 (2010), 113-130.

9. Druţă , S.L. - Classes of general natural almost anti-Hermitian structures on the cotangent bundles, Mediterr. J. Math., 8 (2011), 161-179.

10. Druţă-Romaniuc, S.L. - Quasi-constant holomorphic sectional curvatures of tangent bundles with general natural Kähler structures, An. Ştiinţ. Univ. "Al.I. Cuza" Iaşi. Mat. (N.S.), 58 (2012), 181-193.

11. DruţĂ-Romaniuc, S.-L. - Natural diagonal Riemannian almost product and paraHermitian cotangent bundles, Czechoslovak Math. J., 62 (2012), 937-949.

12. Gadea, P.M.; Masque, J.M. - Classification of almost para-Hermitian manifolds, Rend. Mat. Appl., 11 (1991), 377-396.

13. Gadea, P.M.; Montesinos Amilibia, A. - Spaces of constant para-holomorphic sectional curvature, Pacific J. Math., 136 (1989), 85-101 
14. Heydari, A.; Peyghan, E. - A characterization of the infinitesimal conformal transformations on tangent bundles, Bull. Iranian Math. Soc., 34 (2008), 59-70

15. Ivanov, S.; Zamkovoy, S. - Parahermitian and paraquaternionic manifolds, Differential Geom. Appl., 23 (2005), 205-234.

16. KolÁr, I.; Michor, P.W.; SlovÁk, J. - Natural Operations in Differential Geometry, Springer-Verlag, Berlin, 1993.

17. Kowalski, O.; Sekizawa, M. - Natural transformations of Riemannian metrics on manifolds to metrics on tangent bundles. A classification, Bull. Tokyo Gakugei Univ., 40 (1988), 1-29.

18. Mok, K.P.; Patterson, E.M.; Wong, Y.C. - Structure of symmetric tensors of type $(0,2)$ and tensors of type $(1,1)$ on the tangent bundle, Trans. Amer. Math. Soc., 234 (1977), 253-278.

19. Munteanu, M.I. - CR-structures on the unit cotangent bundle and Bochner type tensor, An. Ştiinţ. Univ. "Al.I. Cuza" Iaşi. Mat. (N.S.), 44 (1998), 125-136 (1999).

20. Oproiu, V.; Papaghiuc, N.; Mitric, G. - Some classes of para-Hermitian structures on cotangent bundles, An. Ştiinţ. Univ. "Al. I. Cuza" Iaşi. Mat. (N.S.), 43 (1997), 7-22 (1998).

21. Oproiu, V.; Poroşniuc, D.D. - A Kähler Einstein structure on the cotangent bundle of a Riemannian manifold, An. Ştiinţ. Univ. "Al. I. Cuza" Iaşi. Mat. (N.S.), 49 (2003), 399-414. (2004).

22. Oproiu, V.; Poroşniuc, D.D. - A class of Kähler Einstein structures on the cotangent bundle, Publ. Math. Debrecen, 66 (2005), 457-478.

23. Peyghan, E.; Heydari, A. - A class of locally symmetric para-Kähler Einstein structures on the cotangent bundle, Int. Math. Forum, 5 (2010), 145-153.

24. Prvanović, M. - Holomorphically projective transformations in a locally product space, Math. Balkanica, 1 (1971), 195-213.

25. Salimov, A.A.; AgCA, F. - Some properties of Sasakian metrics in cotangent bundles, Mediterr. J. Math., 8 (2011), 243-255.

26. Yano, K.; Ishinara, S. - Tangent and Cotangent Bundles: Differential Geometry, Pure and Applied Mathematics, 16, Marcel Dekker, Inc., New York, 1973.

Received: 1.VIII.2012

Accepted: 3.XII.2012
Universitatea Tehnică "Gheorghe Asachi" din Iaşi, Seminarul Matematic, Univ. "Al.I. Cuza", Bd. Carol I, No. 1, 700506-Iaşi, ROMANIA

simonadruta@yahoo.com 